\title{
Conformal Slit Mapping and Its Applications to Brain Surface Parameterization
}

\author{
Yalin Wang ${ }^{1,2}$, Xianfeng Gu ${ }^{3}$, Tony F. Chan ${ }^{2}$, Paul M. Thompson ${ }^{1}$, \\ and Shing-Tung $\mathrm{Yau}^{4}$ \\ ${ }^{1}$ Lab. of Neuro Imaging, UCLA School of Medicine, Los Angeles, CA 90095, USA \\ ylwang@math.ucla.edu \\ 2 Mathematics Department, UCLA, Los Angeles, CA 90095, USA \\ ${ }^{3}$ Comp. Sci. Department, SUNY at Stony Brook, Stony Brook, NY 11794, USA \\ ${ }^{4}$ Department of Mathematics, Harvard University, Cambridge, MA 02138, USA
}

\begin{abstract}
We propose a method that computes a conformal mapping from a multiply connected mesh to the so-called slit domain, which consists of a canonical rectangle or disk in which 3D curved landmarks on the original surfaces are mapped to concentric or parallel lines in the slit domain. In this paper, we studied its application to brain surface parameterization. After cutting along some landmark curve features on surface models of the cerebral cortex, we obtain multiple connected domains. By computing exact harmonic one-forms, closed harmonic one-forms, and holomorphic one-forms, we are able to build a circular slit mapping that conformally maps the surface to an annulus with some concentric arcs and a rectangle with some slits. The whole algorithm is based on solving linear systems so it is very stable. In the slit domain parameterization results, the feature curves are either mapped to straight lines or concentric arcs. This representation is convenient for anatomical visualization, and may assist statistical comparisons of anatomy, surface-based registration and signal processing. Preliminary experimental results parameterizing various brain anatomical surfaces are presented.
\end{abstract}

\section{Introduction}

In this paper, we introduce a new method to conformally map a multiply connected domain to an annulus with multiple concentric arcs (called the circular slit map) or to a rectangle with multiple straight lines (the parallel slit map). It is a global conformal parameterization method without segmentation. First, it computes exact harmonic one-forms and closed harmonic one-forms. Secondly, it computes all bases of holomorphic one-forms. Given appropriate boundary conditions, it can compute a unique circular slit map up to a rotation around the center. The slit mapping computes the intrinsic structure of the given surface, which can be reflected in the shape of the target domain.

Most of brain conformal parameterization methods [1/2/3/4/5]6 can handle the complete brain cortex surface, but can not deal with cortex surfaces with boundaries. The holomorphic flow segmentation method [7] can match cortex surfaces with boundaries or landmarks, but the resulting mappings have singularities, which are very error-prone in practice. Only the Ricci flow method [8]

D. Metaxas et al. (Eds.): MICCAI 2008, Part I, LNCS 5241, pp. 585 593, 2008.

(C) Springer-Verlag Berlin Heidelberg 2008 
and slit map method can handle surfaces with complicated topologies (boundaries and landmarks) without singularities. The Ricci flow method is a nonlinear optimization process, which is much more time consuming than slit map method. The Ricci flow method also has higher requirements for the quality for the tessellations of the surfaces. Because the Ricci flow method involves a lot of transcendental computations, it is not as stable as slit map method. Slit map method is a linear method, which is more efficient and robust.

\subsection{Related Work}

Brain surface parameterization has been studied intensively. Schwartz et al. 9, and Timsari and Leahy [10] computed quasi-isometric flat maps of the cerebral cortex. Drury et al. [1] presented a multiresolution method for flattening the cerebral cortex. Hurdal and Stephenson [1] reported a discrete mapping approach that uses circle packings to produce "flattened" images of cortical surfaces on the sphere, the Euclidean plane, and the hyperbolic plane. The maps obtained are quasi-conformal approximations of classical conformal maps. Haker et al. 2 implemented a finite element approximation for parameterizing brain surfaces via conformal mappings. They select a point on the cortex to map to the north pole of the Riemann sphere and conformally map the rest of the cortical surface to the complex plane by stereographic projection of the Riemann sphere to the complex plane. Gu et al. [3] proposed a method to find a unique conformal mapping between any two genus zero manifolds by minimizing the harmonic energy of the map. They demonstrate this method by conformally mapping a cortical surface to a sphere. Ju et al. 4] presented a least squares conformal mapping method for cortical surface flattening. Joshi et al. [5] proposed a scheme to parameterize the surface of the cerebral cortex by minimizing an energy functional in the $p^{t h}$ norm. Ju et al. 6] reported the results of a quantitative comparison of FreeSurfer [12], CirclePack, and least squares conformal mapping (LSCM) with respect to geometric distortion and computational speed. Wang et al. 7] have used holomorphic 1-forms to parameterize anatomical surfaces with complex (possibly branching) topology. Wang et al. 8] introduced a brain surface conformal mapping algorithm based on algebraic functions. By solving the Yamabe equation with the Ricci flow method, it can conformally map a brain surface to a multi-hole disk.

\section{Theoretical Background}

Suppose $S$ is a surface embedded in $\mathbb{R}^{3}$, with induced Euclidean metric g. $S$ is covered by an atlas $\left\{\left(U_{\alpha}, \phi_{\alpha}\right)\right\}$. Suppose $\left(x_{\alpha}, y_{\alpha}\right)$ is the local parameter on the chart $\left(U_{\alpha}, \phi_{\alpha}\right)$. We say $\left(x_{\alpha}, y_{\alpha}\right)$ is isothermal, if the metric has the representation $\mathbf{g}=e^{2 \lambda\left(x_{\alpha}, y_{\alpha}\right)}\left(d x_{\alpha}^{2}+d y_{\alpha}^{2}\right)$.

The Laplace-Beltrami operator is defined as

$$
\Delta_{\mathrm{g}}=\frac{1}{e^{2 \lambda\left(x_{\alpha}, y_{\alpha}\right)}}\left(\frac{\partial^{2}}{\partial x_{\alpha}^{2}}+\frac{\partial^{2}}{\partial y_{\alpha}^{2}}\right) .
$$

A function $f: S \rightarrow \mathbb{R}$ is harmonic, if $\Delta_{\mathrm{g}} f \equiv 0$. 
Suppose $\omega$ is a differential one-form with the representation $f_{\alpha} d x_{\alpha}+g_{\alpha} d y_{\alpha}$ in the local parameters $\left(x_{\alpha}, y_{\alpha}\right)$, and $f_{\beta} d x_{\beta}+g_{\beta} d y_{\beta}$ in the local parameters $\left(x_{\beta}, y_{\beta}\right)$. Then

$$
\left(\begin{array}{ll}
\frac{\partial x_{\alpha}}{\partial x_{\beta}} & \frac{\partial y_{\alpha}}{\partial x_{\beta}} \\
\frac{\partial x_{\alpha}}{\partial y_{\beta}} & \frac{\partial y_{\alpha}}{\partial y_{\beta}}
\end{array}\right)\left(\begin{array}{c}
f_{\alpha} \\
g_{\alpha}
\end{array}\right)=\left(\begin{array}{c}
f_{\beta} \\
g_{\beta}
\end{array}\right) .
$$

$\omega$ is a closed one-form, if on each chart $\left(x_{\alpha}, y_{\alpha}\right), \frac{\partial f}{\partial y_{\alpha}}-\frac{\partial g}{\partial x_{\alpha}}=0 . \omega$ is an exact one-form, if it equals the gradient of some function. An exact one-form is also a closed one-form. If a closed one-form $\omega$ satisfies $\frac{\partial f}{\partial x_{\alpha}}+\frac{\partial g}{\partial y_{\alpha}}=0$, then $\omega$ is a harmonic one-form. The gradient of a harmonic function is an exact harmonic one-form.

The so-called Hodge star operator turns a one-form $\omega$ to its conjugate ${ }^{*} \omega$, ${ }^{*} \omega=-g_{\alpha} d x_{\alpha}+f_{\alpha} d y_{\alpha}$.

A holomorphic one-form is a complex differential form $\omega+\sqrt{-1}$, $\omega$, where $\omega$ is a harmonic one-form.

Suppose $S$ is an open surface with $n$ boundaries $\gamma_{1}, \cdots, \gamma_{n}$. We can uniquely find a holomorphic one-form $\omega$, such that

$$
\int_{\gamma_{k}} \omega=\left\{\begin{array}{lr}
2 \pi & k=1 \\
-2 \pi & k=2 \\
0 & \text { otherwise }
\end{array}\right.
$$

Definition 1 (Circular Slit Mapping). Fix a point $p_{0}$ on the surface, for any point $p \in S$, let $\gamma$ be an arbitrary path connection $p_{0}$ and $p$, then the circular slit mapping is defined as $\phi(p)=e^{\int_{\gamma} \omega}$.

Theorem 1. The function $\phi$ effects a one-to-one conformal mapping of $M$ onto the annulus $1<|z|<e^{\lambda_{0}}$ minus $n-2$ concentric arcs situated on the circles $|z|=e^{\lambda_{i}}, i=1,2, \cdots, n-2$.

The proof of the above theorem on slit mapping can be found in [13]. For a given choice of the inner and outer circle, the circular slit mapping is uniquely determined up to a rotation around the center. The parallel slit mapping can be defined in a similar way.

Definition 2 (Parallel Slit Mapping). Let $\bar{S}$ be the universal covering space of the surface $S, \pi: \bar{S} \rightarrow S$ be the projection and $\bar{\omega}=\pi^{*} \omega$ be the pull back of $\omega$. Fix a point $\bar{p}_{0}$ on $\bar{S}$, for any point $p \in \bar{S}$, let $\bar{\gamma}$ be an arbitrary path connection $\bar{p}_{0}$ and $\bar{p}$, then the parallel slit mapping is defined as $\bar{\phi}(\bar{p})=\int_{\bar{\gamma}} \bar{\omega}$.

\section{Algorithm Pipeline}

Suppose the input mesh has $n+1$ boundaries, $\partial M=\gamma_{0}-\gamma_{1}-\cdots-\gamma_{n}$. Without loss of generality, we map $\gamma_{0}$ to the outer circle of the circular slit domain, $\gamma_{1}$ to the inner circle, and all the others to the concentric slits.

The algorithm pipeline is as follows :

1 Compute the basis for all exact harmonic one-forms;

2. Compute the basis for all closed harmonic one-forms; 
3. Compute the basis for all holomorphic one-forms;

4. Construct the slit mapping.

\subsection{Basis for Exact Harmonic One-Forms}

The first step of the algorithm is to compute the basis for exact harmonic oneforms. Let $\gamma_{k}$ be an inner boundary, we compute a harmonic function $f_{k}: S \rightarrow \mathbb{R}$ by solving the following Dirichlet problem on the mesh $M:\left\{\begin{array}{l}\Delta f_{k} \equiv 0 \\ \left.f_{k}\right|_{\gamma_{j}}=\delta_{k j}\end{array}\right.$, where $\delta_{k j}$ is the Kronecker function, $\Delta$ is the discrete Laplace-Beltrami operator using the co-tangent formula proposed in [14.

The exact harmonic one-form $\eta_{k}$ can be computed as the gradient of the harmonic function $f_{k}, \eta_{k}=d f_{k}$, and $\left\{\eta_{1}, \eta_{2}, \cdots, \eta_{n}\right\}$ form the basis for the exact harmonic one-forms .

\subsection{Basis for Harmonic One-Forms}

After getting the exact harmonic one-forms, we will compute the closed oneform basis. Let $\gamma_{k}(k>0)$ be an inner boundary. Compute a path from $\gamma_{k}$ to $\gamma_{0}$, denote it as $\zeta_{k}$. $\zeta_{k}$ cut the mesh open to $M_{k}$, while $\zeta_{k}$ itself is split into two boundary segments $\zeta_{k}^{+}$and $\zeta_{k}^{-}$in $M_{k}$. Define a function $g_{k}: M_{k} \rightarrow \mathbb{R}$ by solving a Dirichlet problem,

$$
\left\{\begin{array}{l}
\Delta g_{k} \equiv 0 \\
\left.g_{k}\right|_{\zeta_{k}^{+}}=1 \\
\left.g_{k}\right|_{\zeta_{k}^{-}}=0
\end{array}\right.
$$

Compute the gradient of $g_{k}$ and let $\tau_{k}=d g_{k}$, then map $\tau_{k}$ back to $M$, where $\tau_{k}$ becomes a closed one-form. Then we need to find a function $h_{k}: M \rightarrow \mathbb{R}$, by solving the following linear system: $\Delta\left(\tau_{k}+d h_{k}\right) \equiv 0$.

Updating $\tau_{k}$ to $\tau_{k}+d h_{k}$, we now have $\left\{\tau_{1}, \tau_{2}, \ldots, \tau_{n}\right\}$ as a basis set for all the closed but non-exact harmonic one-forms.

With both the exact harmonic one-form basis and the closed non-exact harmonic one-form basis computed, we can construct the harmonic one-form basis by taking the union of them: $\left\{\eta_{1}, \eta_{2}, \cdots, \eta_{n}, \tau_{1}, \tau_{2}, \cdots, \tau_{n}\right\}$.

\subsection{Basis for Holomorphic One-Forms}

In Step 1 we computed the basis for exact harmonic one-forms $\left\{\eta_{1}, \cdots, \eta_{n}\right\}$. Now we compute their conjugate one-forms $\left\{{ }^{*} \eta_{1}, \cdots,{ }^{*} \eta_{n}\right\}$, so that we can combine all of them together into a holomorphic one-form basis set.

First of all, for $\eta_{k}$ we compute an initial approximation $\eta_{k}^{\prime}$ by a brute-force method using the Hodge star. That is, rotating $\eta_{k}$ by $90^{\circ}$ about the surface normal to obtain $\eta_{k}^{\prime}$. In practice such an initial approximation is usually not accurate enough. In order to improve the accuracy, we employ a technique utilizing the harmonic one-form basis we just computed. From the fact the $\eta_{k}$ is harmonic, we can conclude that its conjugate ${ }^{*} \eta_{k}$ should also be harmonic. 
Therefore, ${ }^{*} \eta_{k}$ can be represented as a linear combination of the base harmonic one-forms: ${ }^{*} \eta_{k}=\sum_{i=1}^{n} a_{i} \eta_{i}+\sum_{i=1}^{n} b_{i} \tau_{i}$.

Using the wedge product $\wedge$, we can construct the following linear system,

$$
\int_{M}{ }^{*} \eta_{k} \wedge \eta_{i}=\int_{M} \eta_{k}^{\prime} \wedge \eta_{i}, \int_{M}{ }^{*} \eta_{k} \wedge \tau_{j}=\int_{M} \eta_{k}^{\prime} \wedge \tau_{j} .
$$

We solve this linear system to obtain the coefficients $a_{i}$ and $b_{i}(i=1,2, \cdots, n)$ for the conjugate one-form ${ }^{*} \eta_{k}$. Pairing each base exact harmonic one-form in the basis with its conjugate, we get a basis set for the holomorphic one-form group on $M:\left\{\eta_{1}+\sqrt{-1}^{*} \eta_{1}, \cdots, \eta_{n}+\sqrt{-1} * \eta_{n}\right\}$

\subsection{Construct Slit Mapping}

After computing the holomorphic one-form basis, we need to find a special holomorphic one-form $\omega=\sum_{i=1}^{n} \lambda_{i}\left(\eta_{i}+\sqrt{-1} * \eta_{i}\right)$, such that the imaginary part of its integral satisfies

$$
\operatorname{Im}\left(\int_{\gamma_{k}} \omega\right)= \begin{cases}-2 \pi & k=1 \\ 0 & k>1\end{cases}
$$

To get the coefficients $\lambda_{i}$, we solve the following linear system for $\lambda_{i}, i=$ $1, \cdots, n$ :

$$
\left(\begin{array}{cccc}
\alpha_{11} & \alpha_{12} & \cdots & \alpha_{1 n} \\
\alpha_{21} & \alpha_{22} & \cdots & \alpha_{2 n} \\
\vdots & \vdots & \ddots & \vdots \\
\alpha_{n 1} & \alpha_{n 2} & \cdots & \alpha_{n n}
\end{array}\right)\left(\begin{array}{c}
\lambda_{1} \\
\lambda_{2} \\
\vdots \\
\lambda_{n}
\end{array}\right)=\left(\begin{array}{c}
-2 \pi \\
0 \\
\vdots \\
0
\end{array}\right)
$$

where $\alpha_{k j}=\int_{\gamma_{j}}{ }^{*} \eta_{k}$.

It can be proven that this linear system has a unique solution, which reflects the fact that $\gamma_{1}$ is mapped to the inner circle of the circular slit domain. Further, the system implies the following equation $\lambda_{1} \alpha_{01}+\lambda_{2} \alpha_{02}+\cdots+\lambda_{n} \alpha_{0 n}=2 \pi$, which means that $\gamma_{0}$ is mapped to the outer circle in the circular slit domain.

After computing the desired holomorphic one-form $\omega$, we are ready to generate the circular slit mapping. What we need to compute is a complex-valued function $\phi: M \rightarrow \mathbb{C}$ by integrating $\omega$ and taking the exponential map. Choosing a base vertex $v_{0}$ arbitrarily, and for each vertex $v \in M$ choosing the shortest path $\gamma$ from $v_{0}$ to $v$, we can compute the map as the following: $\phi(v)=e^{\int_{\gamma} \omega}$.

Based on the circular slit map $\phi$ we just computed, we can compute a parallel slit map $\tau: M \rightarrow \mathbb{C}: \tau(v)=\ln \phi(v)$.

\section{Experimental Results}

We applied our algorithms to parameterize various anatomical surfaces extracted from 3D MRI scans of the brain. In this paper, the segmentations are regarded as given, and result from automated and manual segmentations detailed in our prior work. Figure 1 shows an example of our computation results. For the 


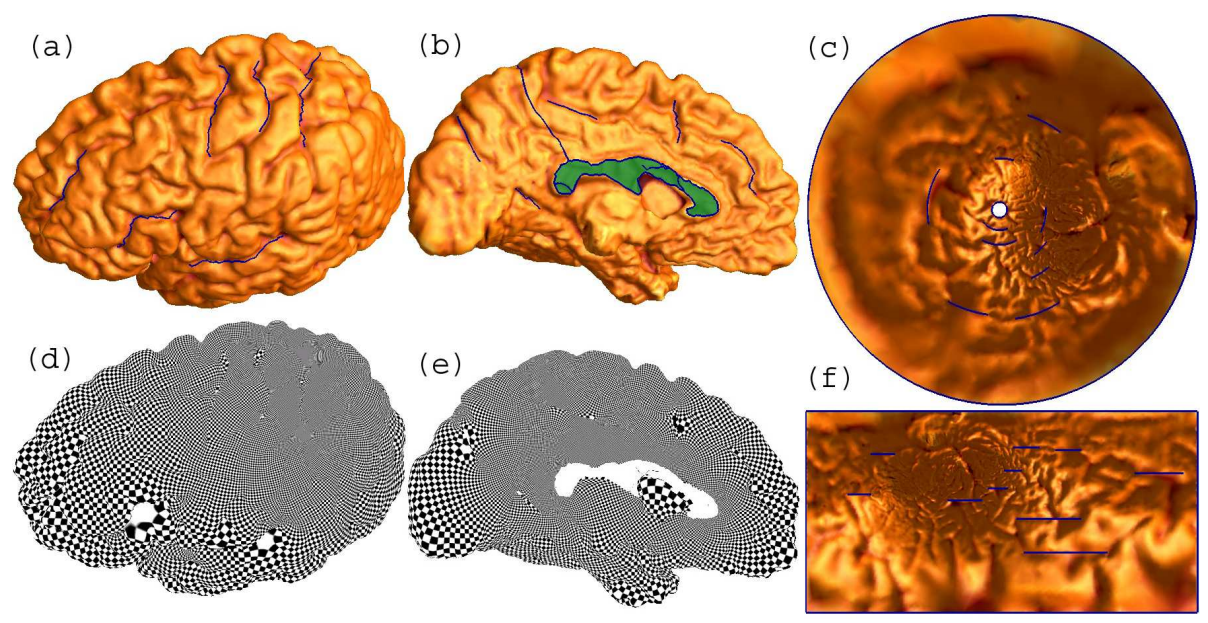

Fig. 1. (a) and (b) show the cortical surface with 12 landmarks cut open, including an open boundary at the corpus callosum (in green); (c) is the parallel slit map result; (f) is the circular slit map result; (d) and (e) show the conformal texture parameterized by the circular slit map (f)

cortical surface of the brain, we cut it open along twelve major landmarks. (a) and (b) show the brain surface from two different views. With our slit mapping algorithm, we can conformally map it to a rectangular domain with four slits (c) and an annulus with ten concentric $\operatorname{arcs}(\mathrm{d})$. (d) and (e) demonstrate the conformal texture mapping as the pull-back of the coordinates induced by the circular slit mapping of (f).

We also tested our algorithm on a left hippocampal surface, a key structure in the medial temporal lobe of the brain, for which parametric shape models are commonly developed for tracking shape differences and longitudinal atrophy in disease. The results are shown in the first row of Subfigure 1 in Figure 2 We leave two holes on the front and back of the hippocampal surface, representing its anterior junction with the amygdala and its posterior limit as it turns into the white matter of the fornix. We also randomly selected two curves lying in regions of high curvature, which are of interest for surface registration research (these could also be boundaries of the CA fields, or other architectonic boundaries, if high-field images are available). The parallel slit mapping result is shown with appropriate landmark curves labeled. We also applied our algorithm to lateral ventricular surface (second row in Subfigure 1 of Figure 2). We introduced three cuts. The motivation for these cuts are based on the topology of the lateral ventricles, in which several horns are joined together at the "atrium" or "trigone". In the parallel slit mapping result, two boundaries are mapped to left and right boundaries, respectively. The rectangle's lengthy aspect ratio reflects its intrinsically long horn-like shape. The third column shows the 


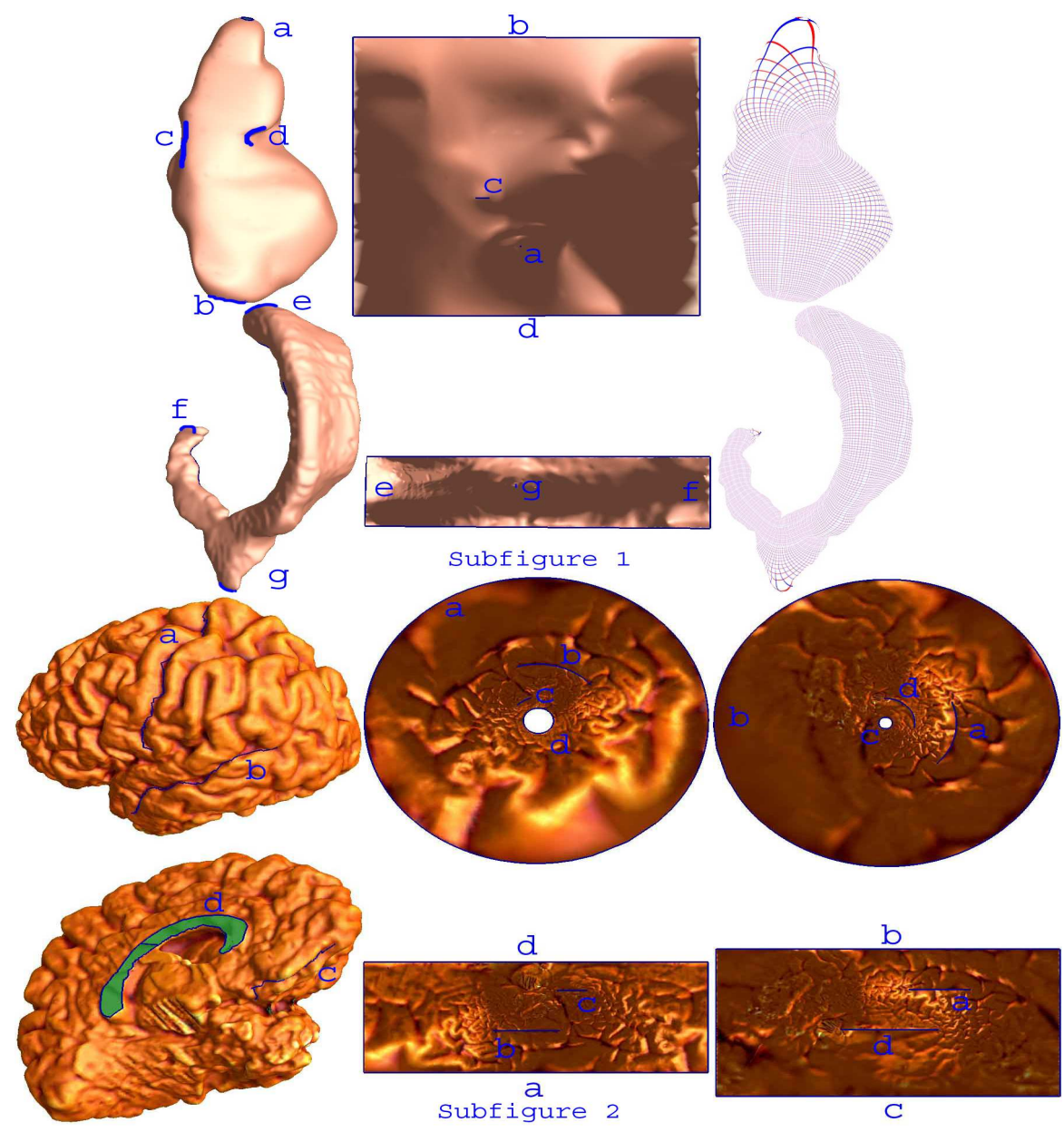

Fig. 2. Subfigure 1 illustrations the parallel slit mapping results for a hippocampal surface and a left lateral ventricle. Surfaces are cut open along various landmarks which are the blue curves on two pictures in the left column. Similar to previous other work, the landmarks are curves are either at the end of shape or follow significant curvatures. In the first row, four landmarks are cut open on a hippocampal surface. In the parallel slit map result, landmarks $b$ and $d$ are mapped to the upper and lower boundaries, respectively. On the second row, three landmarks are introduced on a lateral ventricle surface. In the parallel slit map, e and $f$ are mapped to the left and right boundaries, respectively. Their conformal texture are also shown. In Subfigure 2,conformal parameterization results are shown with different boundary conditions. The first column shows a cerebral cortical surface is cut open along four major sulcal landmarks. The second column shows the circular slit map and parallel slit map results when a pair of landmarks are selected as boundaries landmark a as the exterior circular boundary and $d$ as the inner circular boundary. The third column shows results the other pair of landmarks are selected as boundaries. 
conformal texture mapping as pull-back of the coordinates induced by the slit mapping.

In Figure 2, Subfigure 2 demonstrates various parallel slit mapping results given different boundary conditions. As shown in Subfigure 2, four landmarks were cut open. After the cut, the surface turns into an open boundary genus three surface. For the Equation [1, we selected two different pairs of landmarks as the exterior and inner boundaries by putting the integration of different $\gamma_{k}$ as $2 \pi$ and $-2 \pi$. The second column shows the parameterization results when we use landmark a and $\mathrm{d}$ as the exterior and inner circular boundaries, respectively. The third column shows the parameterization results when we select the other pair of landmark curves as the boundary conditions.

\section{Conclusions and Future Work}

In this paper, we presented a brain surface conformal parameterization method based on the slit mapping, which transfers cortical geometry and any embedded landmarks into a canonical domain, with conformal coordinates. With fixed boundary conditions, our algorithm can compute unique circular slit maps and parallel slit maps, where the positions and lengths of the slits are determined by the conformal equivalence class of the surface. We tested our algorithm on hippocampal, lateral ventricular and cerebral cortical surfaces. Our future work will include empirical application of the slit mapping algorithm to biomedical applications in computational anatomy, including the detection of population differences and the tracking of brain change over time.

\section{References}

1. Hurdal, M.K., Stephenson, K.: Cortical cartography using the discrete conformal approach of circle packings. NeuroImage 23, S119-S128 (2004)

2. Angenent, S., Haker, S., Tannenbaum, A., Kikinis, R.: Conformal geometry and brain flattening. Med. Image Comput. Comput.-Assist. Intervention, 271-278 (September 1999)

3. Gu, X., Wang, Y., Chan, T.F., Thompson, P.M., Yau, S.T.: Genus zero surface conformal mapping and its application to brain surface mapping. IEEE TMI 23(8), 949-958 (2004)

4. Ju, L., Stern, J., Rehm, K., Schaper, K., Hurdal, M.K., Rottenberg, D.: Cortical surface flattening using least squares conformal mapping with minimal metric distortion. In: IEEE ISBI, Arlington, VA, USA, pp. 77-80 (2004)

5. Joshi, A.A., Leahy, R.M., Thompson, P.M., Shattuck, D.W.: Cortical surface parameterization by p-harmonic energy minimization. In: IEEE ISBI, Arlington, VA, USA, pp. 428-431 (2004)

6. Ju, L., Hurdal, M.K., Stern, J., Rehm, K., Schaper, K., Rottenberg, D.: Quantitative evaluation of three surface flattening methods. NeuroImage 28(4), 869-880 (2005)

7. Wang, Y., Lui, L.M., Gu, X., Hayashi, K.M., Chan, T.F., Toga, A.W., Thompson, P.M., Yau, S.T.: Brain surface conformal parameterization using Riemann surface structure. IEEE TMI 26(6), 853-865 (2007) 
8. Wang, Y., Gu, X., Chan, T.F., Thompson, P.M., Yau, S.T.: Brain surface conformal parameterization with algebraic functions. In: Larsen, R., Nielsen, M., Sporring, J. (eds.) MICCAI 2006, Part II. LNCS, vol. 4191, pp. 946-954. Springer, Heidelberg (2006)

9. Schwartz, E., Shaw, A., Wolfson, E.: A numerical solution to the generalized mapmaker's problem: Flattening nonconvex polyhedral surfaces. IEEE TPAMI 11(9), 1005-1008 (1989)

10. Timsari, B., Leahy, R.M.: An optimization method for creating semi-isometric flat maps of the cerebral cortex. In: Proceedings of SPIE, Medical Imaging, San Diego, CA (February 2000)

11. Drury, H.A., Van Essen, D.C., Anderson, C.H., Lee, C.W., Coogan, T.A., Lewis, J.W.: Computerized mappings of the cerebral cortex: A multiresolution flattening method and a surface-based coordinate system. J. Cognitive Neurosciences 8, 1-28 (1996)

12. Fischl, B., Sereno, M.I., Dale, A.M.: Cortical surface-based analysis II: Inflation, flatteningm and a surface-based coordinate system. NeuroImage 9, 179-194 (1999)

13. Ahlfors, L.V.: Complex Analysis. McGraw-hill, New York (1953)

14. Pinkall, U., Polthier, K.: Computing discrete minimal surfaces and their conjugate. Experimental Mathematics 2(1), 15-36 (1993) 